\title{
Sensory neuropathy due to RFC1 in a patient with ALS: more than a coincidence?
}

\author{
Florian Schoeberl ${ }^{1,2}$ - Angela Abicht ${ }^{3,4} \cdot$ Clemens Kuepper $^{1} \cdot$ Stefanie Voelk $^{1} \cdot$ Stefan Sonnenfeld $^{1}$. \\ Matthias Tonon ${ }^{1} \cdot$ Annalisa Schaub ${ }^{4} \cdot$ Veronika Scholz $^{4} \cdot$ Stephanie Kleinle $^{4} \cdot$ Hannes Erdmann ${ }^{4} \cdot$ Dieter A. Wolf $^{4}$. \\ Peter Reilich ${ }^{1,3}$
}

Received: 12 August 2021 / Revised: 28 September 2021 / Accepted: 29 September 2021 / Published online: 25 November 2021

(c) The Author(s) 2021

Dear Sirs,

In 2019, non-parametric linkage analyses and genome sequencing revealed that biallelic AAGGG expansions in the replication factor $\mathrm{C}$ subunit 1 (RFCl) gene are a frequent cause of late-onset ataxia [1]. Subsequent studies described the phenotypic spectrum of patients with pathological $R F C 1$ expansion: they mainly presented in their fifth decade of life with a triad of cerebellar dysfunction (i.e., gait ataxia, dysarthria, ocular motor disorders), sensory neuropathy with concomitant sensory ataxia, and vestibular areflexia bilaterally, denoted by the acronym CANVAS [1]. Quite recently, a multicentre observational study has shown that $R F C l$ expansion comprises a multisystemic disease with a chronic dry cough, dysautonomia, and bradykinesia as additional clinical features of variable degree [2].

It is still an outstanding issue, whether biallelic AAGGG expansion in $R F C l$ are not associated with an even broader phenotypic spectrum of neurodegenerative diseases.

A 64-year-old male presented with a 1-year history of progressive and painless weakness of both hands. Neurological examination revealed generalized polytopic muscle fasciculations in 4/4 levels, muscle paresis for finger adduction/

Florian Schoeberl and Angela Abicht have contributed equally to this work.

Florian Schoeberl

Floian.Schoeberl@med.uni-muenchen.de

1 Department of Neurology, Ludwig-Maximilians-University, Marchioninistr. 15, 81377 Munich, Germany

2 German Center for Vertigo and Balance Disorders, DSGZ, Ludwig-Maximilians-University, Munich, Germany

3 Department of Neurology, Friedrich-Baur-Institut, Neuromuscular Center, Ludwig-Maximilians-University, Munich, Germany

4 Medical Genetics Centre, Munich, Germany abduction (r: MRC 4-5, 1: MRC 4), finger extension (r: MRC 4-5, 1: MRC 4), thumb opposition (r: MRC 4-5, 1: MRC 4), wrist extension/flexion (r: MRC 4-5, 1: MRC 4) and hip flexion ( $r / 1$ : MRC 4-5). A split hand sign was conspicuous on both sides. Muscle reflexes were brisk on the left upper limb with decreased ankle jerks bilaterally. There were neither relevant sensory/proprioceptive deficits nor clinical signs of ataxia/vestibulopathy. Clinical suspicion of a degenerative motor neuron disease was confirmed by electromyography, muscle ultrasound and transcranial magnetic stimulation (for details see Table 1). Sural and superficial peroneal nerve potentials and sensory evoked potentials of the tibial nerves (P40) were absent bilaterally.

Finally, amyotrophic lateral sclerosis (ALS) was diagnosed according to the current diagnostic criteria (see Table 1). Additionally, regarding sensory nerve conduction studies and evoked potentials subclinical sensory neuropathy/neuronopathy was diagnosed.

Acquired conditions for sensory neuropathies/neuronopathies were excluded (see Table 1). The patient did not consent to a recommended additional CSF analysis.

Genetic analysis by CRISPR/Cas9 target enrichment and Oxford Nanopore long-read sequencing [3], revealed biallelic AAGGG repeat expansions ( 400) of the RFC1 locus. Negative results of all genetic testing are listed in Table 1.

Due to the detected biallelic $R F C 1$ repeat expansions we post-hoc performed vestibular testing by inner ear calorics and video-assisted head-impulse-test, which revealed isolated bilateral presbyvestibulopathy in the low-frequency range (see Table 1).

This case with a diagnosis of ALS, additional subclinical sensory neuro(no)pathy and bilateral presbyvestibulopathy in the low-frequency range in association with a biallelic $R F C 1$ expansion raises the following noteworthy future question: is ALS/motor neuron disease within the phenotypic spectrum of biallelic $R F C l$ repeat expansions? 
Table 1 An overview of the diagnostic procedures and findings in our patient

\begin{tabular}{|c|c|}
\hline Diagnostic test & Result \\
\hline Muscle ultrasound & Polytopic muscle fasciculations in $4 / 4$ levels \\
\hline EMG & Acute and chronic denervation in $4 / 4$ levels \\
\hline Transcranial magnetic stimulation & $\begin{array}{l}\text { Delayed central motor latency and reduced ampli- } \\
\text { tude to the left abductor pollicis brevis; normal } \\
\text { central motor latencies and amplitudes to the } \\
\text { right abductor pollicis brevis and both tibial } \\
\text { anterior muscles }\end{array}$ \\
\hline
\end{tabular}

Neurofilament light chain serum levels (SIMOA)

Gold Coast criteria (2020)

Awaji-Shima criteria (2008)

Sensory nerve conduction studies

Motor nerve conduction studies

Sensory evoked potentials

Caloric irrigation inner ear $\left(\right.$ warm $/$ cold water $\left.^{\circ}\right)$

Video-assisted head impulse-test (median gain at $60 \mathrm{~ms}$ )

MRI-scan brain (3 T)

MRI-scan cervical spine (3 T)

MRI-scan brachial plexus (3 T)

Additional laboratory testings
Interpretation

Abnormal

Abnormal

Abnormal

$82 \mathrm{pg} / \mathrm{ml}$ (limit value for ALS: $<45 \mathrm{pg} / \mathrm{ml}$ )

Progressive motor impairment, documented by history or repeated clinical assessment, preceded by normal motor function

Upper and lower motor neuron dysfunction in at least one body region or lower motor neuron dysfunction in at least two body regions

Investigation findings that excluded alternative disease processes

Probable ALS: clinical and electrophysiological signs of lower motor neuron degeneration in at least two regions

Absent potentials of both sural and superficial peroneus nerves; normal potentials of median and ulnar nerves

Reduced amplitudes of both median and ulnar and Abnormal left-sided tibial and peroneus nerves; normal potentials of right-sided tibial and peroneus nerves

Absent P40 of both tibial nerves; normal N9 and N20 of both median nerves

Right: warm $-4.8^{\circ}$, Cold $5.7^{\circ}$

Left: warm $5.6^{\circ}$, Cold $-7.7^{\circ}$

Lying in the range of bilateral presbyvestibulopathy (i.e. $6^{\circ}-25^{\circ}$ )

Right: $0.93 \pm 0.11$

Left: $0.97 \pm 0.06$

No pyramidal tract lesion, no brainstem pathology, no cerebellar atrophy, no frontal cortex atrophy

No spinal cord stenosis, no spinal cord lesions, no Unremarkable nerve root compressions

No lesions, no increased contrast-enhancement, Unremarkable no thickened nerve fascicles

Serum glucose, HbA1c-level, liver enzymes, creatinine, vitamin B12 pathway, anti-neuronal antibodies (anti-Hu, -Ri, -Yo, -Ma2, -Tr, Amphiphysin), monoclonal proteins, gangliosideantibodies (anti-GM1, -GM2, -GD1a, -GD1b, -GQ1b) anti-MAG, antinuclear antibody subtypes (anti-SS-A, -SS-B, -Sm, -RNP, -Scl70, -PmScl, -Jo1), anti-neutrophilic cytoplasmic antibodies, ganglionic acetylcholine receptor antibodies
Increased

Fulfilled

Fulfilled

Abnormal

Abnormal

Abnormal

Unremarkable

Unremarkable

Unremarkable 
Table 1 (continued)

\begin{tabular}{|c|c|c|}
\hline Diagnostic test & Result & Interpretation \\
\hline Genetic testings & $\begin{array}{l}\text { biallelic AAGGG repeat expansions ( 400) of the } \\
\text { RFC1 locus } \\
\text { NGS-based gene panel testing (ANXA11, } \\
\text { CHCHD10, EPHA4, FUS, HNRNPA1, KIF5A, } \\
\text { NEK1, OPTN, PFN1, SOD1, TARDBP, TBK1, } \\
\text { UBQLN2, UNC13A, VAPB, VCP) did not reveal } \\
\text { variants of unknown significance, pathogenic } \\
\text { or likely pathogenic variants (ACMG class 3, } \\
4 \text { or 5) } \\
\text { Testing for repeat expansions in C9orf72 (FTD/ } \\
\text { ALS), ATXN1 (SCA1), ATXN2 (SCA2), ATXN3 } \\
\text { (SCA3), and HTT (Huntington Disease) did } \\
\text { not reveal any expansion in the pathological or } \\
\text { intermediate range: } \\
C 9 \text { orf72 (repeat units allele 1/2): } 8 / 8 \\
\text { ATXN1 (repeat units allele } 1 / 2): 28 / 29 \\
\text { ATXN2 (repeat units allele } 1 / 2): 22 / 22 \\
\text { ATXN3 (repeat units allele } 1 / 2): 14 / 30 \\
\text { HTT (repeat units allele } 1 / 2): 21 / 27\end{array}$ & $\begin{array}{l}\text { Pathological RFC1 repeat expansion } \\
\text { ALS gene panel unremarkable } \\
\text { Repeat expansions in C9orf72, ATXN1, } \\
\text { ATXN2, ATXN3, and HTT unremark- } \\
\text { able }\end{array}$ \\
\hline
\end{tabular}

To our knowledge, this is the first case of an ALS patient with a concomitant subclinical sensory neuro(no) pathy and bilateral presbyvestibulopathy carrying a biallelic $R F C 1$ repeat expansion. The number of genes associated with monogenic forms or increased risk of ALS is constantly growing including intermediate expansions of the SCA 1,2 genes and huntingtin-trinucleotide expansions $[4,5]$. Acknowledging previous reports with abnormal findings in sensory nerve conduction studies in up to $20 \%$ of patients with ALS [6] and earlier morphological findings in sensory nerve biopsies suggesting loss of sensory root ganglion neurons [7], an involvement of pathological $R F C l$ expansions as additional monogenic form or at least genetic risk factor for ALS might be discussed. However, one must admit, that we cannot differentiate an association of pathological RFCl expansions with a combined phenotype of ALS and sensory neuro(no)pathy in our patient from a bare coincidence of ALS with a beginning CANVAS phenotype due to $R F C 1$ pathology. A recent study indeed found $R F C l$ expansions exclusively in so far "idiopathic" sensory neuropathies, but not in patients with "idiopathic" sensorimotor neuropathies [8]. Thus a clear link of RFC1 pathology with the motor system is missing so far. Since RFCl mediated pathology affects the sensory ganglion cells and not the peripheral sensory nerves, the findings from Currò et al. are plausible [8]. A recent study revealed that pathogenic SPTLC1 mutations are not only associated with the phenotype of sensory and autonomic neuropathy (i.e. HSAN type 1), but also with juvenile onset ALS [9]. And, for the rare syndrome of "facial onset sensory motor neuronopathy" (FOSMN) typically beginning with sensory symptoms of the trigeminal nerves, underlying TDP-43 pathology in sensory ganglion cells as well as motor neurons was confirmed [10-12], thus classifying FOSMN currently as a rare form of motor neuron disease.

An important limitation of the presented case is, that we cannot assess the influence of rare genetic variants with small effect size or their combinatory effect in terms of polygenic risk modification.

In conclusion, the presented case with a concomitant sensory neuro(no)pathy and proven $R F C l$ expansion in addition to ALS should prompt a more systematical search for RFC1 expansion in larger patient cohorts with ALS and unexplained sensory involvement in order to disentangle a possible role of RFCl pathology in ALS.

Author contributions All authors contributed to the study conception and design. The first draft of the manuscript was written by FS and all authors commented on previous versions of the manuscript. All authors read and approved the final manuscript.

Funding Open Access funding enabled and organized by Projekt DEAL. No funding was received for this study.

\section{Declarations}

Conflicts of interest Dr. Schöberl reports no disclosures; Dr. Abicht reports no disclosures; Dr. Kuepper reports no disclosures; Dr. Voe$1 \mathrm{k}$ reports no disclosures; Dr. Sonnenfeld reports no disclosures; Dr. Tonon reports no disclosures; Ms Schaub reports no disclosures; Ms Scholz reports no disclosures; Dr. Kleinle reports no disclosures; Dr. Wolf reports no disclosures; Dr. Erdmann reports no disclosures; Dr. Reilich reports no disclosures.

Ethical approval We have obtained the patient's permission and informed consent for publishing of his information/case. 
Open Access This article is licensed under a Creative Commons Attribution 4.0 International License, which permits use, sharing, adaptation, distribution and reproduction in any medium or format, as long as you give appropriate credit to the original author(s) and the source, provide a link to the Creative Commons licence, and indicate if changes were made. The images or other third party material in this article are included in the article's Creative Commons licence, unless indicated otherwise in a credit line to the material. If material is not included in the article's Creative Commons licence and your intended use is not permitted by statutory regulation or exceeds the permitted use, you will need to obtain permission directly from the copyright holder. To view a copy of this licence, visit http://creativecommons.org/licenses/by/4.0/.

\section{References}

1. Cortese A, Tozza S, Yau W et al (2020) Cerebellar ataxia, neuropathy, vestibular areflexia syndrome due to RFC1 repeat expansion. Brain J Neurol [online serial]. https://pubmed.ncbi.nlm.nih. gov/32040566/. Accessed 4 July 2021

2. Traschütz A, Cortese A, Reich S et al (2021) Natural history, phenotypic spectrum, and discriminative features of multisystemic RFC1 disease. Neurology [online serial]. https://pubmed.ncbi. nlm.nih.gov/33495376/. Accessed 4 July 2021

3. Gilpatrick T, Lee I, Graham JE et al (2020) Targeted nanopore sequencing with Cas9-guided adaptor ligation. Nat Biotechnol 38:433-438

4. Li P, Sun X, Xia G et al (2016) ATXN2-AS, a gene antisense to ATXN2, is associated with spinocerebellar ataxia type 2 and amyotrophic lateral sclerosis. Ann Neurol [online serial]. https:// pubmed.ncbi.nlm.nih.gov/27531668/. Accessed 4 July 2021
5. Dewan R, Chia R, Ding J et al (2021) Pathogenic huntingtin repeat expansions in patients with frontotemporal dementia and amyotrophic lateral sclerosis. Neuron [online serial]. https://pubmed. ncbi.nlm.nih.gov/33242422/. Accessed 4 July 2021

6. Pugdahl K, Fuglsang-Frederiksen A, de Carvalho M et al (2007) Generalised sensory system abnormalities in amyotrophic lateral sclerosis: a European multicentre study. J Neurol Neurosurg Psychiatry 78:746-749

7. Heads T, Pollock M, Robertson A, Sutherland W, Allpress S (1991) Sensory nerve pathology in amyotrophic lateral sclerosis. Acta Neuropathol (Berl) [online serial]. https://pubmed.ncbi.nlm. nih.gov/1662002/. Accessed 15 July 2021

8. Currò R, Salvalaggio A, Tozza S et al (2021) RFC1 expansions are a common cause of idiopathic sensory neuropathy. Brain J Neurol [online serial]. 2021. https://pubmed.ncbi.nlm.nih.gov/33969391/. Accessed 26 Sept 2021

9. Jo J, R C, De M et al (2021) Association of variants in the SPTLC1 gene with juvenile amyotrophic lateral sclerosis. JAMA Neurol [online serial]. https://pubmed.ncbi.nlm.nih.gov/34459 874/. Accessed 26 Sept 2021

10. Vucic S, Tian D, Chong PST, Cudkowicz ME, Hedley-Whyte ET, Cros D (2006) Facial onset sensory and motor neuronopathy (FOSMN syndrome): a novel syndrome in neurology. Brain 129:3384-3390

11. Vucic S (2014) Facial onset sensory motor neuronopathy (FOSMN) syndrome: an unusual amyotrophic lateral sclerosis phenotype? J Neurol Neurosurg Psychiatry [online serial]. https:// pubmed.ncbi.nlm.nih.gov/24643461/. Accessed 26 Sept 2021

12. Rossor A, Jaunmuktane Z, Rossor M, Hoti G, Reilly M (2019) TDP43 pathology in the brain, spinal cord, and dorsal root ganglia of a patient with FOSMN. Neurology [online serial]. https:// pubmed.ncbi.nlm.nih.gov/30700593/. Accessed 26 Sept 2021 